\title{
Loss of function of SVBP leads to autosomal recessive intellectual disability, microcephaly, ataxia, and hypotonia
}

\author{
Zafar lqbal, $\mathrm{PhD}^{1,2}$, Hasan Tawamie, $\mathrm{PhD}^{3,4}$, Wei $\mathrm{Ba}, \mathrm{PhD}^{1}$, André Reis, Professor $\mathrm{MD}^{3}$, \\ Bassam Al Halak, MD ${ }^{5}$, Heinrich Sticht, Professor PhD ${ }^{6}$, Steffen Uebe, PhD ${ }^{3}$, Nael Nadif Kasri, PhD ${ }^{1}$, \\ Sheikh Riazuddin, Professor PhD ${ }^{7,8}$, Hans van Bokhoven, Professor PhD ${ }^{1}$ and Rami Abou Jamra, MD ${ }^{3,4}$
}

Purpose: Identifying and characterizing novel causes of autosomal recessive intellectual disability based on systematic clinical and genetic evaluation, followed by functional experiments.

Methods: Clinical examinations, genome-wide positional mapping, and sequencing were followed by quantitative polymerase chain reaction and western blot of the protein SVBP and its interaction partners. We then knocked down the gene in rat primary hippocampal neurons and evaluated the consequences on synapses.

Results: We identified a founder, homozygous stop-gain variant in $\operatorname{SVBP}\left(\mathrm{c} .82 \mathrm{C}>\mathrm{T} ; \mathrm{p}\right.$.[Gln $28^{*}$ ]) in four affected individuals from two independent families with intellectual disability, microcephaly, ataxia, and muscular hypotonia. SVBP encodes a small chaperone protein that transports and stabilizes two angiogenesis regulators, VASH1 and VASH2. The altered protein is unstable and nonfunctional since transfected HeLa cells with mutant SVBP did not reveal evidence for immunoreactive SVBP protein fragments and cotransfection with VASH1 showed a severe reduction of VASH1 in medium and cell lysate. Knocking down $S v b p$ in rat primary hippocampal neurons led to a significant decrease in the number of excitatory synapses.

Conclusion: SVBP is not only involved in angiogenesis, but also has vital functions in the central nervous system. Biallelic loss-offunction variants in $S V B P$ lead to intellectual disability.

Genetics in Medicine (2019) 21:1790-1796; https://doi.org/10.1038/s41436018-0415-8

Keywords: CCD23; VASH1; intellectual disability; NGS; hippocampal neurons

\section{INTRODUCTION}

Intellectual disability (ID) is one of the most common reasons for referral to clinical genetic centers and has impact on the affected individuals, their families, and the health system. ${ }^{1,2}$ It has a broad spectrum of severity, disease course, and associated symptoms. Despite huge progress in the past few years, a considerable number of affected individuals remain undiagnosed after physical examinations and genetic tests, such as copy-number variant (CNV) analysis, targeted gene sequencing, and exome or even genome sequencing. ${ }^{1,3}$ Given the high contribution of de novo pathogenic variants to the prevalence of ID in Western populations, chromosomal microarrays and exome sequencing analyses of child-parent trios have proven to be a powerful strategy to uncover causative CNVs and nucleotide variants in up to $60 \%$ of all patients. ${ }^{3-6}$ This is still not the case for autosomal recessive forms of ID (ARID). Recent large-scale studies have shown that the combination of comprehensive clinical evaluation, genome-wide mapping, exome sequencing, and sharing phenotypes and genetic variants with other scientists represent the most successful strategy to identify ID genes. ${ }^{7-12}$ Despite all the recent success, number of undiagnosed ARID patients is still high. Reasons for this include the large number of genes anticipated to underlie ARID, the small family sizes in which these conditions occur, and the difficulty to assign causality for homozygous or compound heterozygous gene variants. ${ }^{2,6,13}$

Here, we report the identification of a founder homozygous variant in SVBP (HGNC:29204) in four individuals from two families with intellectual disability. We also present functional characterization of the variant and the small vasohibin binding protein (SVBP).

\footnotetext{
${ }^{1}$ Department of Human Genetics, Donders Institute for Brain, Cognition and Behaviour, Radboud university medical centerNijmegen, The Netherlands; ${ }^{2}$ Department of Neurology, Oslo University Hospital, Oslo, Norway; ${ }^{3}$ Institute of Human Genetics, Friedrich-Alexander-Universität Erlangen-Nürnberg, Erlangen, Germany; ${ }^{4}$ Institute of Human Genetics, University Medical Center Leipzig, Leipzig, Germany; ${ }^{5}$ Praxis of Pediatrics, Kefrenbel, Idlib, Syrian Arab Republic; ${ }^{6}$ Bioinformatics, Institute of Biochemistry, Friedrich-AlexanderUniversität Erlangen-Nürnberg, Erlangen, Germany; ${ }^{7}$ Center for Genetic Diseases, Shaheed Zulfiqar Ali Bhutto Medical University, Pakistan Institute of Medical Sciences, Islamabad, Pakistan; ${ }^{8}$ Allama Iqbal Medical College, University of Health Sciences, Lahore, Pakistan. Correspondence: Hans Bokhoven (Hans.vanBokhoven@radboudumc.nl) or Rami Abou Jamra (rami.aboujamra@medizin.uni-leipzig.de)

Shared first authors: Zafar Iqbal, Hasan Tawamie, Wei Ba

These authors contributed equally: Hans van Bokhoven, Rami Abou Jamra
} 


\section{MATERIALS AND METHODS}

\section{Ethics statement}

This study was approved by the ethic committees of the University of Bonn and of the University of Erlangen-Nuremberg in Germany, the Institutional Review Board (IRB) of the Centre of Excellence in Molecular Biology (CEMB), University of the Punjab, Lahore, Pakistan, as well as the ethics committee of the Radboud university medical center, Nijmegen, the Netherlands. Informed consent of all examined persons or their guardians was obtained, including consent to publish photos.

\section{Genotyping, CNV analysis, and homozygosity mapping}

$\mathrm{CNV}$ analysis was performed by genotyping affected individuals with high-resolution genotyping techniques using $6 \mathrm{k}$ Illumina (Illumina, San Diego, CA) and 6.0 Affymetrix singlenucleotide polymorphism (SNP) arrays (Affymetrix, Santa Clara, CA). For CNV detection, the SNP array data was analyzed by using Copy Number Analyzer for GeneChip (CNAG) (Affymetrix, Santa Clara, CA). The regions of homozygosity of at least $1 \mathrm{Mb}$ were determined either by visual inspection or by using Homozygosity Mapper (http:// www.homozygositymapper.org/).

\section{Exome sequencing}

For the Syrian family, enrichment for exome sequencing of individual V-3 was performed on DNA using the SureSelect Human All Exon Kit V2 (50 Mb) (Agilent Technologies, Santa Clara, CA, USA). Posthybridization barcodes were used to allow for multiplexing (Agilent Technologies). The Beckman Coulter SPRIworks platform (Beckman Coulter, Danvers, MA, USA) was used for the automated library preparation. Sequencing was carried out with 70-bp pairedend reads on a SOLiD5500XL system (Life Technologies, Carlsbad, CA, USA). On average, more than 160 million reads were obtained. Read mapping to the hg19 reference genome was performed with SOLiD LifeScope software v2.5. Variant calling was performed using the LifeScope software v2.5 using high-stringency settings and GATK v1.4 (ref. ${ }^{14}$ ) after local realignment of indels. All called variants up to the size of 20 bp were evaluated. Only variants meeting the following criteria were considered for further analyses: (1) a minor allele frequency (MAF) of $<1 \%$ in the Exome Variant Server (ESP, version ESP6500, accessed December 2014), in the 1000 Genomes Project (1000 G, version 1000G2012, access July 2014), and in-house exomes (>600 exomes); (2) exonic (nonsynonymous and indels) or located at splice sites $(+/-20 \mathrm{bp})$; and (3) minimum of $5 \times$ coverage. Analyses focused on the candidate regions, but eventually all inheritance patterns were considered. The variants were then prioritized according to in silico parameters (annotation using ANNOVAR ${ }^{15}$ ). Polymerase chain reaction (PCR) and Sanger sequencing were performed using standard protocols for the exclusion of technical artifacts and for segregation testing. Details of the sequencing and filtering procedures are also provided elsewhere. ${ }^{16}$ Average target coverage of 121 was obtained, and $85 \%$ of the targeted sequences were covered to a depth of $5 \times$ or more, which is sufficient to identify homozygous variants. A total of 45,373 single-nucleotide variants (SNVs) and 2894 insertions or deletions were identified.

For the Pakistani family, targeted next-generation sequencing (NGS) was used to sequence the complete identified homozygous regions on one-quarter plate of a Roche 454 as described previously. ${ }^{13}$ In brief, all coding and noncoding exons of respective homozygous regions (Supplementary Table 1) including surrounding sequences of $20 \mathrm{bp}$ to cover the splice sites and untranslated regions were targeted on a $385 \mathrm{~K}$ sequence capture array (Roche NimbleGen, Madison, WI). Sequence capture, amplification of captured DNA, sequencing, and data analysis were done as described previously. $^{13,17}$ Variant prioritization was performed as described previously. ${ }^{17}$ Briefly, variants were selected on the basis of MAF $<1 \%$, exonic and canonical splice sites, gene function, and nucleotide conservation with a phyloP score $>2.5$ (ref. $^{18}$ ).

\section{RT-PCR}

Total RNA was extracted using RNeasy Mini Kit (Qiagen, Hilden, Germany) from lymphoblastoid cell lines of the affected individuals V-3 and V-5 of the Syrian family, their mother, and healthy brother, as well as of six healthy controls from the same geographical area and the same lymphoblastoid cell lines charge. Reverse transcription PCR (RT-PCR) was carried out using a Superscript II RT system according to manufacturer's instructions (Life Technologies, Carlsbad, CA). Quantitative PCR (qPCR) was performed using SYBR Green PCR Master Mix (Life Technologies, Carlsbad, CA) and the following specific primers: (SVBP_exprF1: 5'-ACTGCTGCTGCTCCAGTTC-3'; SVBP_expR1: 5'-AAG GCCAAACAGAAATCAGC-3') and (SVBP_exprF2: 5'-GG AGGCTCCACCTTCCAGT-3'; SVBP_expR2: 5'-GTGGA TCCATGGCTTGACTT-3'). Adjustment and standardization were achieved based on control PCR products using specific primers for $H T P R 1, T B P$, and $B 2 M$. All quantitative PCR reactions were performed in an Applied Biosystems QuantStudio 12 Flex real-time PCR machine (Applied Biosystems, Grand Island, NY).

\section{Western blotting}

First, we cloned SVBP (ENST00000372521) and VASH1 into pcDNA-Flag and cDNA ${ }^{\mathrm{m}} 3.1$ myc-His vectors, respectively, using a QIAgene infusion kit and $\mathrm{pCR}^{\circledR} \mathrm{II}-\mathrm{TOPO}$ cloning systems (Qiagen, Hilden, Germany). The transfection of HeLa cells with constructed plasmids was performed using polyethyleneimine (PEI). Two days after the transfection, cells were lysed in $1 \%$ sodium dodecyl sulfate (SDS) cell lysis buffer consisting of $10 \mathrm{mM}$ Tris- $\mathrm{HCl}, \mathrm{pH} 7.5,150 \mathrm{mM} \mathrm{NaCl}, 1 \%$ SDS supplemented with $0.5 \mathrm{mM}$ phenylmethylsulfonyl fluoride, and Complete EDTA-free Protease Inhibitor Cocktail (Roche Diagnostics, Risch-Rotkreuz, Switzerland). The medium was concentrated 50-fold using an Amicon Ultra-15 
a

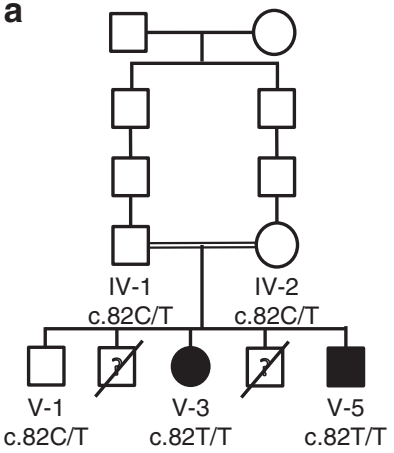

V-3

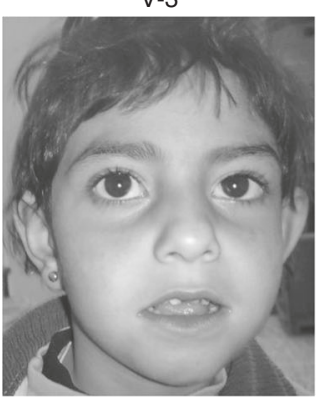

V-5

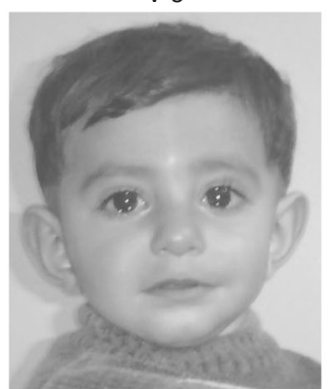

b

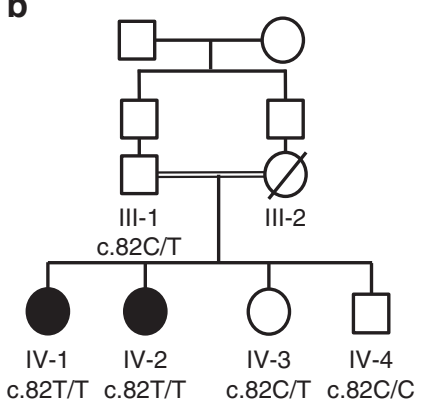

IV-1

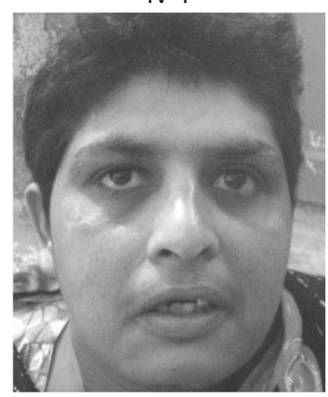

IV-2

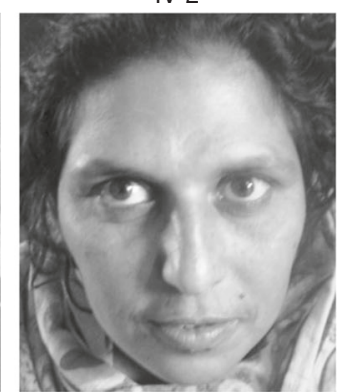

Fig. 1 Pedigrees and photographs of the affected individuals. Pedigrees including genotypes and photographs of the examined siblings of the Syrian (a) and Pakistani (b) families, respectively.

(10,000 MWCO; Millipore). Equal amounts of protein from cell lysates and concentrated medium were separated by SDSpolyacrylamide gel electrophoresis (nuSDS-PAGE) and 2-(Nmorpholino)ethanesulfonic acid (MES) buffer and then transferred onto nitrocellulose membranes (GE Healthcare Europe, Freiburg, Germany). Blotting was performed according to standard procedures. The primary antibodies were antihuman VASH1 $\mathrm{mAb}^{19}$ and antiflag tag (Abcam, Cambridge, United Kingdom). Normalization was performed using antihuman histone $3 \mathrm{H} 3 \mathrm{mAb}$ and $\beta$-actin. Immunoreactive protein bands were detected using SuperSignal West Femto Reagents (Thermo Fisher Scientific, Waltham, Massachusetts, United States) and Amersham hyperfilm ECL LAS4000 .

\section{Svbp knockdown in rat hippocampal neurons}

Hippocampal neuron cultures were prepared from embryonic day 18 rats (Taconic, Sprague Dawley, Charles River) and infected with lentiviruses expressing green fluorescent protein (GFP) and short hairpin RNA (shRNA) as described previously. ${ }^{20}$ Briefly, hippocampal tissue was separated and meninges were removed carefully with forceps under the dissecting microscope. Tissues were digested with $0.25 \%$ trypsin (Corning) for 15 minutes before dissociation. Culture media contained $1 \mu \mathrm{g} / \mathrm{ml}$ fungizone (amphotericin B, Gibco) and $1 \mu \mathrm{M}$ Ara-C (Sigmam, St. Louis, MO, USA) until harvest. Cultures were examined at 14 days in vitro (DIV14) enumerating the numbers of excitatory synapses in the infected GFP positive cells. To reduce the expression of endogenous $S V B P$ in the hippocampal neuron cells, shRNAs targeting the coding region of $S V B P$ were designed. Scrambled
shRNA, which matches nothing in rodent genomes or transcripts, was used as control. Oligonucleotides were first annealed and ligated into the RNAi-pSuper vector (Clontech, Mountain View, CA) and then cloned into the lentiviral vector pTRIP. $^{20}$ This vector utilizes the human $\mathrm{H} 1$ promoter to control shRNA expression and the cytomegalovirus (CMV) promoter to control GFP expression. The sequences targeted were scramble shRNA, SVBP-sh\#1, SVBP-sh\#2. All constructs were verified by sequencing. Neurons were infected at DIV1 and fixed at DIV14 using 4\% paraformaldehyde in phosphate buffered saline.

\section{Immunocytochemistry and image acquisition}

Fixed neurons were permeabilized with $0.2 \%$ Triton and stained with antisera to VGLUT (1:1000; mouse, Synaptic Systems) and PSD-95 (1:250; Thermo Scientific). Images were taken using a Zeiss LSM 510 Meta Confocal Microscope and a $40 \times$ objective. For each neuron, numbers of juxtaposed VGLUT/PSD-95 puncta were counted per dendritic length, counting at least $200 \mu \mathrm{m}$ of dendritic length/neuron.

\section{RESULTS}

\section{Clinical manifestations}

The first family is a consanguineous family of Syrian descent with one healthy boy and two affected individuals (V-3 and $\mathrm{V}-5$ ) (Fig. 1a). The prenatal period of $\mathrm{V}-3$ was unremarkable. At delivery, she developed cyanosis and was unconscious. In the neonatal period, she had muscular hypotonia and had early closed sutures. She learned walking at the age of three years. At the age of five years she could speak few words and obey easy orders. Furthermore, she presented with 


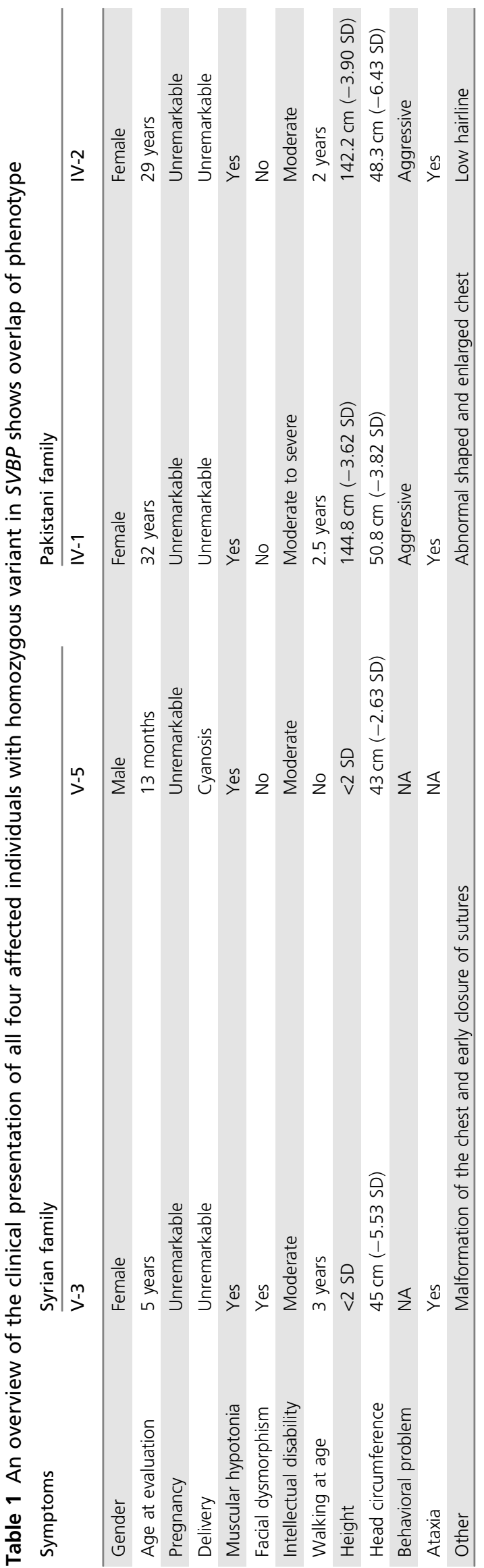

microcephaly (head circumference was $45 \mathrm{~cm}(-5.53 \mathrm{SD})$, Table 1), chest deformity (mild pectus excavatum), mild ataxia with a broad based gait, and growth retardation (however, no exact measurements were available). No magnetic resonance image (MRI) or computed tomography (CT) scans were available. The prenatal period and birth of V5 were unremarkable. His neonatal development was unremarkable and the closure of sutures proceeded normally. However, at the age of 13 months he still could not crawl or stand. He also presented with microcephaly (head circumference was $43 \mathrm{~cm}$ [-2.63 SD], Table 1).

The second family is a consanguineous family of Pakistani descent presenting with two affected girls (IV-1 and IV-2) and two healthy siblings (Fig. 1b). The prenatal period and delivery of both affected members were unremarkable. Clinical examination of both affected individuals at the ages of 29 and 32 years showed intellectual disability with delayed and unclear speech, short stature, microcephaly, muscular hypotonia, ataxia, and aggressive behavior (Table 1). Patient IV-1 had an abnormal-shaped and enlarged chest. The CT scan of both affected individuals showed normal brain structures.

\section{Genetic mapping and variant identification}

Both families were independently analyzed in two large cohort studies, ${ }^{8,10}$ by a combination of homozygosity mapping and next-generation sequencing under the assumption of a homozygous identical by descent causative variant. Genome-wide autozygosity mapping of the Syrian and Pakistani families revealed for each three candidate regions, i.e., homozygous identical by descent genomic stretches, of a total length of $49.8 \mathrm{Mb}$ and $63.5 \mathrm{Mb}$, respectively (Supplementary Table 1). Subsequently, exome sequencing was performed for the affected individual (V-3) of the Syrian family, and targeted NGS of candidate genes was performed for the affected individual (IV-1) of the Pakistani family, as described previously. ${ }^{13,16}$ In both families we were left with a variant in SVBP (also known as CCDC23, NM_199342.3) (c. $82 \mathrm{C}>\mathrm{T}, \mathrm{p}$. [Gln28*]) that is absent in public databases (gnomAD and $1000 \mathrm{G}$ ) and in 380 Syrian and 240 Pakistani healthy controls. In a next step, we have found that the variant is embedded in a shared identical by state haplotype of at least $1.78 \mathrm{Mb}$ between $42,637,613 \mathrm{bp}$ and $44,420,248 \mathrm{bp}$ on chromosome 1 (hg19/GRCh37) (Supplementary Figure 1). Further, segregation analysis was performed including available unaffected members of both families, which revealed segregation of the identified homozygous variant p. $\left(\mathrm{G} \ln 28^{\star}\right)$ with the phenotype in both families (Fig. 1a, b, and Supplementary Figures 2 and 3).

The families did not have further shared, homozygous stretches. The gnomAD database included only a few loss-offunction variants in $S V B P$ and none are homozygous. Further evaluation of the sequencing data of both families, including considering other inheritance patterns, did not reveal further convincing variants that are rare and predicted to have an impact on the protein. 

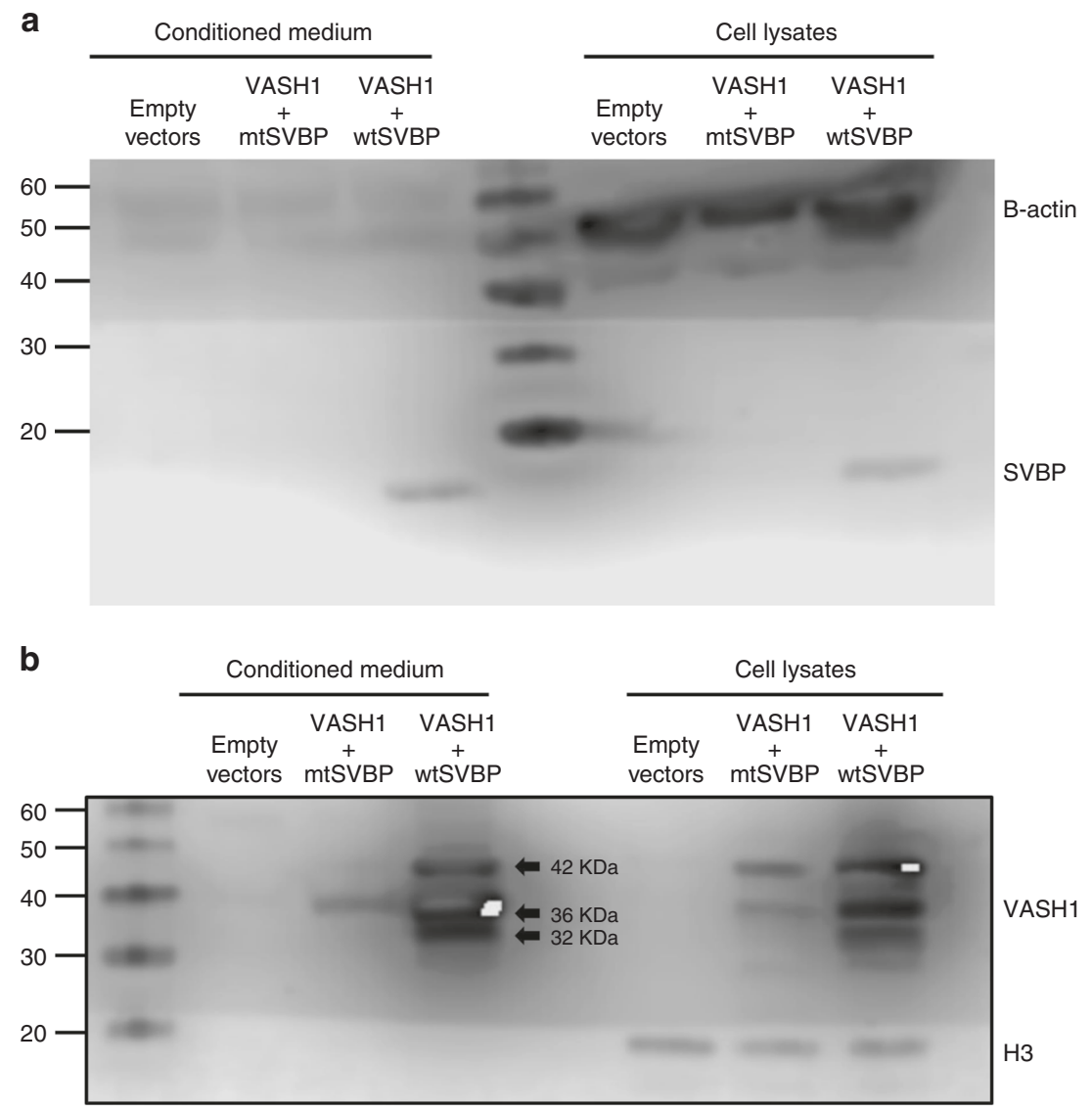

Fig. 2 Pathogenicity of SVBP variant on the VASH1 secretion. The cell lysate and conditioned medium were collected from HeLa cells cotransfected with human VASH1 and either wild type or mutant N-terminal FLAG-SVBP expression vectors $(\mathbf{a}, \mathbf{b})$. Concentrated conditioned medium and the cell lysates were evaluated by western blot $(\mathbf{a}, \mathbf{b})$. Western blot analysis showed that in cells cotransfected with mutant SVBP construct, no altered protein was detected (a) and also the posttranslational forms of VASH1 ( $42 \mathrm{KDa}, 36 \mathrm{KDa}$, and $32 \mathrm{KDa}$ ) are reduced in comparison with the cells cotransfected with the wild type SVBP construct (b). $\beta$-actin and histone $\mathrm{H} 3$ were used as internal controls for the western blot of SVBP and VASH1, respectively.

\section{Premature stop codon SVBP variant impairs the VASH1 secretion and solubility}

The variant in SVBP generates a premature stop codon at position 28 located in penultimate exon 2 of SVBP. Premature stop codons often lead to nonsense-mediated messenger RNA (mRNA) decay (NMD). To assess this hypothesis for the variant that we have identified, $\mathrm{qPCR}$ was performed using total RNA isolated from the lymphoblastoid cell lines of the affected individuals, the mother, and a healthy sibling of the Syrian family, as well as six healthy controls. The results showed high variability in expression levels of SVBP between the individuals, and no significant reduction in the mRNA levels of SVBP in patients (Supplementary Figure 4).

Molecular modeling using the Parcoil tool ${ }^{21}$ revealed that the altered protein would lose its highly conserved coiled-coil domain (CCD) (Supplementary Figure 5). Due to its small size of 28 amino acids, the truncated protein is possibly nonfunctional and/or degraded. To investigate this assumption, we cotransfected HeLa cells with an equimolar amount of human VASH1 and N-terminal flag-tagged human SVBP (wild type and mutant) and analyzed the VASH1 and SVBP expression in the cell lysate and conditioned medium using western blot. The results of western blot revealed no evidence for the presence of truncated SVBP in both cell lysate and concentrated, conditioned medium (Fig. 2). Also, a significant reduction in three posttranslational forms of VASH1 (42 $\mathrm{KDa}, 36 \mathrm{KDa}$, and $32 \mathrm{KDa}$ ) was observed in the conditioned medium and cell lysate of the mutant SVBP-transfected cells (Fig. 2).

\section{SVBP functions are associated with excitatory synapse formation in hippocampal neurons}

Synapse dysfunction is a hallmark of intellectual disability. Therefore, we measured the excitatory synapse formation in rat hippocampal neurons in which we knocked down approximately $50 \%$ of $S v b p$ expression using two independent shRNAs (Supplementary Figure 6). At DIV14, we analyzed vesicular glutamate transporter 1 (VGLUT) and postsynaptic density protein 95 (PSD-95), markers for excitatory pre- and postsynapses, respectively. The results revealed that neurons with reduced Svbp expression showed decreased juxtaposed VGLUT/PSD-95 puncta per dendritic length compared with 
a

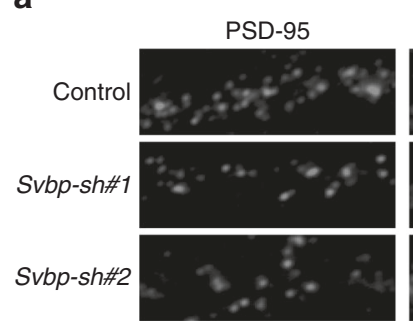

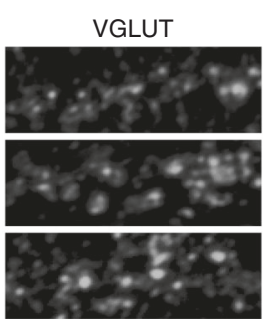

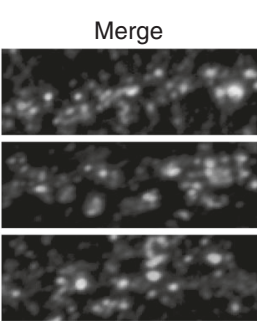

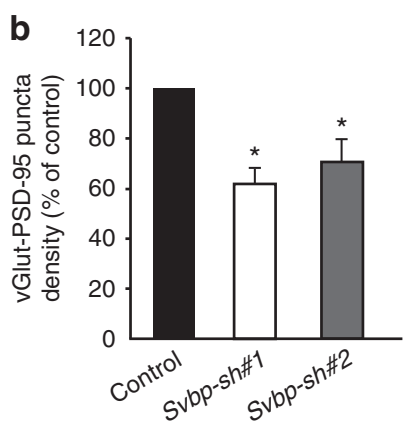

Fig. 3 Svbp knockdown in hippocampal neuron and its impact on excitatory synapse formation. Downregulation of Svbp reduces excitatory synapses in hippocampal primary neurons. a Representative double-label images of PSD-95 (red) and VGLUT (green) in low-density hippocampal neurons. b Quantification of the amount of juxtaposing PSD-95-VGLUT puncta, normalized to control. Control short hairpin RNA (shRNA): $n=12 ;$ Svbp-sh\#1: $n=13$; Svbp-sh\#2: $n=13$. Scale bars, $5 \mu \mathrm{m} ;{ }^{*} P<0.05$, $t$-test; error bars represent SEM.

the controls (Svbp-sh\#1 62\% $\pm 8 \%$ and Svbp-sh\#2 71\% $\pm 9 \%$ as compared with control) (Fig. 3a, b).

\section{DISCUSSION}

Mapping and sequencing revealed the same homozygous premature stop codon variant (c. $\left.82 \mathrm{C}>\mathrm{T}, \mathrm{p} .\left[\mathrm{G} \ln 28^{\star}\right]\right)$ in $S V B P$ in two apparently unrelated and geographically remote consanguineous families from Syria and Pakistan. The affected individuals presented an overlapping phenotype of intellectual disability, microcephaly, and muscular hypotonia. In addition, affected individuals presented ataxia (Syrian patient V-5 was at the time of assessment too young to evaluate the clinical presence of ataxia) (Table 1 ). The affected individuals shared a common haplotype of $1.78 \mathrm{Mb}$ (see Supplementary Figure 1), suggesting a common ancestor of both families. The variant was not reported in control samples. We hypothesized that SVBP is a novel candidate gene for autosomal recessive intellectual disability.

SVBP encodes a highly conserved protein among mammals and is abundantly expressed in testis, tibial nerve, and brain, especially the cerebellum. ${ }^{19}$ On the cellular level, SVBP chaperone protein is produced and accumulates near the surface, where it binds vasohibin proteins VASH1 and VASH2, facilitating their solubility, stability, and secretion out of the cells. This interaction is essential for the angiogenic activity of the vasohibin protein (VASH1 and VASH2) family. ${ }^{19,22}$ These proteins are secreted without classical secretory signal sequences, and are secreted by an unconventional protein secretion (UPS) pathway. Kadonosono et al. recently determined the domain architecture of VASH1, which is required to complex with SVBP and facilitate its secretion to the extracellular space. ${ }^{23}$ Interestingly, two recent papers reported that the VASHs complexed to SVBP exert tubulin tyrosine carboxypeptidase activity that controls the detyronisation of tubulin. ${ }^{24,25}$ Knockdown of vasohibins reduced detyrosinated a-tubulin levels and disrupted neuronal migration in the mouse neocortex during development. ${ }^{24,25}$

We could not prove that the loss-of-function variant in SVBP leads to an accelerated decay of RNA (NMD). However, due to the unreliable qPCR results, NMD cannot be excluded.
Nonetheless, we have proved that it leads to a significant reduction of VASH1 protein levels in the cell lysates and in the cell medium. Truncated SVBP could not be detected by western blots of cells transfected with mutant $S V B P$-expressing constructs, suggesting that it is rapidly degraded. It has already been reported that the full length of VASH1 protein $(44 \mathrm{kDa})$ is posttranslationally edited, resulting in four smaller forms $(42 \mathrm{kDa}, 36 \mathrm{kDa}, 32 \mathrm{KD}$, and $27 \mathrm{kDa}$ ) and that SVBP stabilizes these posttranslational forms of VASH1 by increasing its solubility and inhibiting ubiquitination in the cytosol, eventually enhancing VASH1's extracellular release. ${ }^{19}$ The same study has shown that knocking down the SVBP expression by small interfering RNA (siRNA) decreased the ability to extract VASH1 protein and also decreased its secretion into the medium. ${ }^{19}$ These findings are comparable with our results and we therefore assume that the truncated, unstable SVBP leads to decreased solubility and secretion of VASH1. Thus, we consider this founder variant to be pathogenic and causative if biallelic. The heterozygous parents show no symptoms. This may seem to contradict the results of Suzuki et al., ${ }^{19}$ who showed a dose-dependent effect of SVBP on VASH1. However, biochemical changes or observations in functional analyses in vitro of the heterozygous pathogenic variant do not necessarily mean clinical representations.

We have shown that SVBP knockdown in primary hippocampal neurons leads to a reduction of the number of excitatory synapses (Fig. 3). The balance of excitatory and inhibitory synapse formation is required during the assembly of neural circuitry in the brain. Accordingly, this balance is disrupted in a wide variety of neurodevelopmental disorders, such as ID, autism spectrum disorders, epilepsy, and Rett syndrome. ${ }^{26,27}$ The notable decrease in the excitatory synapse observed in the hippocampal neurons following Svbp knockdown provides the first evidence for a role of SVBP in synapse formation.

Given the clinical and genetic overlapping of the affected individuals, the proven pathogenicity of the variant, our results on the influence of SVBP on synapse formation, and recent literature on the role of vasohibins on neuronal 
migration in mouse, we present here $S V B P$ as a further gene of unspecific intellectual disability with ataxia, muscular hypotonia, and microcephaly.

\section{SUPPLEMENTARY INFORMATION}

The online version of this article (https://doi.org/10.1038/s41436$018-0415-8)$ contains supplementary material, which is available to authorized users.

\section{ACKNOWLEDGEMENTS}

The study was supported by grants from the German Research Foundation (AB393/2-1 and AB393/2-2 to R.A.J.), by grants from DAAD (Deutscher Akademischer Austauschdienst) to H.T., by the European Union's Seventh Framework Program (Gencodys; grant 241995 to H.v.B. and S.R.), and the Netherlands Organization for Health Research and Development (917-96-346 and 907-00-365 to N.N.K.). We thank Kornelia Neveling for targeted NGS experiments in the Pakistani family. We thank all who have helped to complete this work. We also thank the affected individuals and their parents for participating in this study.

\section{DISCLOSURE}

The authors declare no conflict of interest.

Publisher's note: Springer Nature remains neutral with regard to jurisdictional claims in published maps and institutional affiliations.

\section{REFERENCES}

1. Willemsen $\mathrm{MH}$, Kleefstra T. Making headway with genetic diagnostics of intellectual disabilities. Clin Genet. 2014;85:101-110.

2. Musante L, Ropers HH. Genetics of recessive cognitive disorders. Trends Genet. 2014;30:32-39.

3. Vissers LE, Gilissen C, Veltman JA. Genetic studies in intellectual disability and related disorders. Nat Rev Genet. 2016;17:9-18.

4. de Ligt J, Willemsen MH, van Bon BWM, Kleefstra T, Yntema HG, Kroes $\mathrm{T}$. et al. Diagnostic exome sequencing in persons with severe intellectual disability. N Engl J Med. 2012;367:1921-1929.

5. Rauch A, Wieczorek D, Graf E, Wieland T, Endele S, Schwarzmayr T, et al. Range of genetic mutations associated with severe non-syndromic sporadic intellectual disability: an exome sequencing study. Lancet. 2012;380:1674-1682.

6. Deciphering Developmental Disorders Study. Large-scale discovery of novel genetic causes of developmental disorders. Nature. 2015;519:223-228.

7. Najmabadi H, Hu H, Garshasbi M, Zemojtel T, Abedini SS, Chen W, et al. Deep sequencing reveals 50 novel genes for recessive cognitive disorders. Nature. 2011;478:57-63.

8. Riazuddin S, Hussain M, Razzaq A, Iqbal Z, Shahzad M, Polla DL, et al. Exome sequencing of Pakistani consanguineous families identifies 30 novel candidate genes for recessive intellectual disability. Mol Psychiatry. 2017;22:1604-1614.
9. Anazi S, Maddirevula S, Faqeih E, Alsedairy H, Alzahrani F, Shamseldin $\mathrm{HE}$, et al. Clinical genomics expands the morbid genome of intellectual disability and offers a high diagnostic yield. Mol Psychiatry. 2017;22:615-624

10. Reuter MS, Tawamie H, Buchert R, Hosny Gebril O, Froukh T, Thiel C, et al. Diagnostic yield and novel candidate genes by exome sequencing in 152 consanguineous families with neurodevelopmental disorders. JAMA Psychiatry. 2017;74:293-299.

11. Trujillano D, Bertoli-Avella AM, Kumar Kandaswamy K, Weiss ME, Köster J, Marais A, et al. Clinical exome sequencing: results from 2819 samples reflecting 1000 families. Eur J Hum Genet. 2017;25:176-182.

12. Kochinke K, Zweier C, Nijhof B, Fenckova M, Cizek P, Honti F, et al. Systematic phenomics analysis deconvolutes genes mutated in intellectual disability into biologically coherent modules. Am J Hum Genet. 2016;98:149-164.

13. Iqbal $Z$, van Bokhoven $H$. Identifying genes responsible for intellectual disability in consanguineous families. Hum Hered. 2014;77:150-160.

14. McKenna A, Hanna M, Banks E, Sivachenko A, Cibulskis K, Kernytsky A, et al. The Genome Analysis Toolkit: a MapReduce framework for analyzing next-generation DNA sequencing data. Genome Res. 2010;20:1297-1303.

15. Wang $\mathrm{K}, \mathrm{Li} \mathrm{M}$, Hakonarson $\mathrm{H}$. ANNOVAR: functional annotation of genetic variants from high-throughput sequencing data. Nucleic Acids Res. 2010;38:e164.

16. Riecken LB, Tawamie H, Dornblut C, Buchert R, Ismayel A, Schulz A, et al. Inhibition of RAS activation due to a homozygous ezrin variant in patients with profound intellectual disability. Hum Mutat. 2015;36:270-278.

17. Hoischen A, Gilissen C, Arts P, Wieskamp N, van der Vliet W, Vermeer S, et al. Massively parallel sequencing of ataxia genes after array-based enrichment. Hum Mutat. 2010;31:494-499.

18. Vissers LE, de Ligt J, Gilissen C, Janssen I, Steehouwer M, de Vries P . et al. A de novo paradigm for mental retardation. Nat Genet. 2010;42:1109-1112.

19. Suzuki $Y$, Kobayashi M, Miyashita $H$, Ohta $H$, Sonoda $H$, Sato $Y$. Isolation of a small vasohibin-binding protein (SVBP) and its role in vasohibin secretion. J Cell Sci. 2010;123 pt 18:3094-3101.

20. Nadif Kasri N, Nakano-Kobayashi A, Malinow R, Li B, van Aelst L. The Rho-linked mental retardation protein oligophrenin-1 controls synapse maturation and plasticity by stabilizing AMPA receptors. Genes Dev. 2009;23:1289-1302.

21. McDonnell $A V$, Jiang $T$, Keating $A E$, Berger B. Paircoil2: Improved prediction of coiled coils from sequence. Bioinformatics. 2006:22:356-358

22. Xue $X$, Gao $W$, Sun $B, X u Y$, Han $B$, Wang $F$, et al. Vasohibin 2 is transcriptionally activated and promotes angiogenesis in hepatocellular carcinoma. Oncogene. 2013;32:1724-1734.

23. Kadonosono $T$, Yimchuen $W$, Tsubaki $T$, Shiozawa $T$, Suzuki $Y$, Kuchimaru $T$, et al. Domain architecture of vasohibins required for their chaperone-dependent unconventional extracellular release. Protein Sci. 2017:26:452-463.

24. Nieuwenhuis J, Adamopoulos A, Bleijerveld OB, Mazouzi A, Stickel E, Celie $P$, et al. Vasohibins encode tubulin detyrosinating activity. Science. 2017;358:1453-1456.

25. Aillaud C, Bosc C, Peris L, Bosson A, Heemeryck P, van Dijk J, et al. Vasohibins/SVBP are tubulin carboxypeptidases (TCPS) that regulate neuron differentiation. Science. 2017;358:1448-1453.

26. Nelson SB, Valakh V. Excitatory/inhibitory balance and circuit homeostasis in autism spectrum disorders. Neuron. 2015;87:684-698.

27. Tatti R, Haley MS, Swanson OK, Tselha T, Maffei A. Neurophysiology and regulation of the balance between excitation and inhibition in neocortical circuits. Biol Psychiatry. 2017;81:821-831. 